\title{
REVIEW
}

\section{Metallic nanostructures for efficient LED lighting}

\author{
Gabriel Lozano ${ }^{1}$, Said RK Rodriguez ${ }^{2}$, Marc A Verschuuren ${ }^{3}$ and Jaime Gómez Rivas ${ }^{4,5}$
}

Light-emitting diodes (LEDs) are driving a shift toward energy-efficient illumination. Nonetheless, modifying the emission intensities, colors and directionalities of LEDs in specific ways remains a challenge often tackled by incorporating secondary optical components. Metallic nanostructures supporting plasmonic resonances are an interesting alternative to this approach due to their strong light-matter interaction, which facilitates control over light emission without requiring external secondary optical components. This review discusses new methods that enhance the efficiencies of LEDs using nanostructured metals. This is an emerging field that incorporates physics, materials science, device technology and industry. First, we provide a general overview of state-of-the-art LED lighting, discussing the main characteristics required of both quantum wells and color converters to efficiently generate white light. Then, we discuss the main challenges in this field as well as the potential of metallic nanostructures to circumvent them. We review several of the most relevant demonstrations of LEDs in combination with metallic nanostructures, which have resulted in light-emitting devices with improved performance. We also highlight a few recent studies in applied plasmonics that, although exploratory and eminently fundamental, may lead to new solutions in illumination. Light: Science \& Applications (2016) 5, e16080; doi:10.1038/lsa.2016.80; published online 3 June 2016

Keywords: light-emitting diodes; nanophotonics; plasmonics; solid-state lighting

\section{INTRODUCTION}

Solid-state lighting (SSL) is an illumination technology that has emerged in the past decade due to the development of white lightemitting diodes (LEDs). Currently, LEDs use a mature technology that can outperform traditional light sources due to their higher efficiencies, longer lifetimes, fast switching, robustness and compact size ${ }^{1,2}$. The working principle of LEDs is based on electroluminescence, that is, the radiative recombination of injected electron-hole pairs in a material. Electroluminescence in inorganic semiconductors was first observed by Round ${ }^{3}$, who applied a voltage across two contacts on a $\mathrm{SiC}$ crystal to generate yellow light. Electroluminescence was intensely investigated in subsequent years ${ }^{4}$ and was reported in several III-V semiconductors in the $1950 \mathrm{~s}^{5,6}$. Techniques to create $\mathrm{p}-\mathrm{n}$ junctions also improved, leading to the demonstration of infrared and red LED emission in GaAs and GaAsP in $1962^{7,8}$. Although blue LED emission turned out to be more complex than initially thought, due to difficulties associated with producing high-quality GaN and doping this material, it was finally accomplished in the early 1990s by two independent research groups ${ }^{9-11}$. This achievement represented a technological breakthrough that led to the award, in 2014, of the Nobel Prize in Physics to Isamu Akasaki, Hiroshi Amano and Shuji Nakamura. We expect to see widespread replacement of traditional light sources with LEDs within the next two decades, leading to a considerable reduction in worldwide electricity consumption. To facilitate this transition, we must integrate LEDs into many different applications. To do this, we must be able to accurately and specifically control brightness, color and directionality of light emitted from LEDs. It appears that this control may be achieved using nanostructures $^{12}$.

Nanostructures, which have dimensions comparable to the wavelength of light, are especially suited to enhancing light-matter interactions ${ }^{13}$. Metallic surfaces and nanostructures supporting surface plasmon polariton (SPP) resonances are of particular interest in this regard $^{14}$. These resonances have their origin in the coherent oscillation of charge carriers in the metal. The spontaneous emission from sources in the proximity of metals can be modified by SPPs, thereby influencing the emission rate and directionality ${ }^{15-34}$. These modifications are analogous to the resonant amplification and directional radiation of antennas. Therefore, metallic nanoparticles supporting SPPs have been referred to as optical antennas or nanoantennas ${ }^{35,36}$. However, integrating such resonant nanostructures into state-of-theart lighting applications remains challenging. The vast majority of studies has focused on modification of the emission properties of single and/or low-efficiency emitters ${ }^{23,37,38}$, while real applications in SSL require modification of emission over macroscopic areas, typically in the $\mathrm{mm}^{2}$ range, of highly efficient emitters for which the typical photoluminescence quantum yield (QY) exceeds $90 \%$. Until recently, these stringent requirements have limited the use of plasmonic structures for SSL. This situation is quickly changing due to the introduction of cost-effective nanofabrication techniques for use in

\footnotetext{
${ }^{1}$ Instituto de Ciencia de Materiales de Sevilla, Consejo Superior de Investigaciones Científicas-Universidad de Sevilla (CSIC-US), 41092 Sevilla, Spain; ${ }^{2}$ Laboratoire de Photonique et de Nanostructures (LPN), Centre National de la Recherche Scientifique (CNRS), 91460 Marcoussis, France; ${ }^{3}$ Philips Research Laboratories, 5656AE Eindhoven, The Netherlands; ${ }^{4}$ Dutch Institute for Fundamental Energy Research, $5600 \mathrm{HH}$ Eindhoven, The Netherlands and ${ }^{5} \mathrm{COBRA}$ Research Institute, Technical University of Eindhoven, Eindhoven, The Netherlands

Correspondence: G Lozano, Email: g.lozano@csic.es; J Gómez Rivas, Email: j.gomezrivas@differ.nl

Received 2 August 2015; revised 10 December 2015; accepted 25 January 2016; accepted article preview online 26 January 2016
} 
light extraction, spectral shaping of emissions and strong beaming, without requiring additional external optical components ${ }^{37,39-42}$. This article reviews recent developments regarding nanostructured metallic surfaces and nanoantennas for use in SSL, an emerging field that provides new opportunities for plasmonics applications.

\section{SOLID-STATE WHITE-LIGHT GENERATION}

Organic LEDs ${ }^{43-50}$ and light-emitting electrochemical cells ${ }^{51,52}$ are lightweight, flat and thin large-area diffuse light sources that represent new illumination technologies. However, inorganic LEDs are currently best suited for general illumination purposes ${ }^{53}$. Therefore, the following discussion focuses on inorganic LEDs. There are two generic approaches to generating artificial white light using inorganic semiconductor LEDs ${ }^{54-56}$. In the first, several LEDs emit different colors that are combined to produce white light ${ }^{57}$. Although significant advances have been made in the development of nanoscale LEDs ${ }^{58-62}$, the maximum efficiency of all semiconductor-based white LEDs is limited by the relatively low efficiencies of green and yellow LEDs, a challenge referred to as the green-yellow gap. In the second and currently the most prevailing approach, highly efficient blue LEDs are used to generate green and red light via color conversion using one or more photoluminescent materials, traditionally called phosphors ${ }^{63-65}$. This second approach is illustrated in Figure 1. The phosphor must have the following: (i) a close-to-one QY to maximize blue-to-green/ red conversion efficiency; (ii) excellent temperature and chemical stabilities; (iii) moderate thermal quenching of emissions at temperatures over $100^{\circ} \mathrm{C}$; (iv) an absorption spectrum that overlaps with the blue LED emission spectrum; (v) a large absorption cross section; and (vi) an emission spectrum that leads to high-quality white-light emission. Therefore, one of the greatest remaining challenges in SSL is the difficulty associated with simultaneously tuning the chemical, structural and optical characteristics of the phosphor material that fulfills the above requirements. This challenge has become the subject of extensive research in materials science ${ }^{66-73}$.

Currently, the leading commercial methodology for obtaining white light using phosphors, so-called phosphor-converted LEDs (pcLEDs), uses yttrium aluminum garnet $\left(\mathrm{YAG}, \mathrm{Y}_{3} \mathrm{Al}_{5} \mathrm{O}_{12}\right.$ ) doped with $\mathrm{Ce}^{3+}$ rare-earth ions (YAG:Ce) as the phosphor. Phosphors based on rareearth ions have low absorption coefficients in the blue region, resulting in the need for a relatively large amount of material (the typical thickness of such phosphors is on the order of several tens of microns). Such phosphor layers incorporate randomly positioned light scatterers that maximize light extraction ${ }^{74-76}$, albeit with a

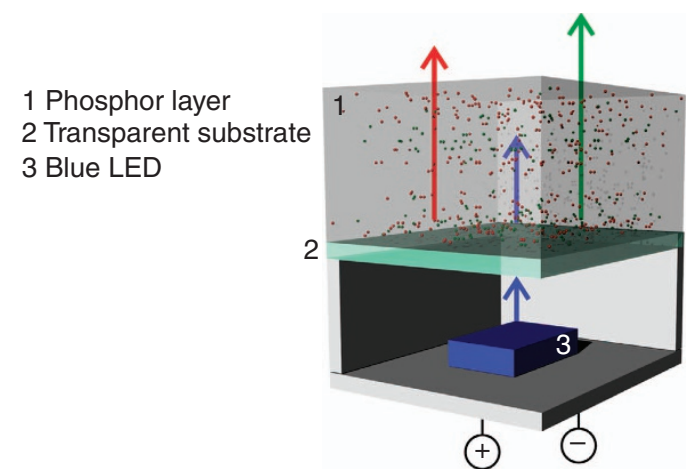

Figure 1 Scheme depicting a pcLED. An electrically biased LED emits blue light, which excites the phosphor material. The mixing of the emission from the phosphor and the non-absorbed blue light is perceived as white light by the human eye.
Lambertian angular profile. Moreover, approximately half of the converted light exits the phosphor layer in the backward direction, that is, toward the blue LED. For these reasons, additional optical elements, such as mirrors or scattering materials, are incorporated into the device to maximize light output.

\section{Efficiency of LEDs}

In general terms, the efficiency of a light-emitting device is the product of three partial efficiencies:

$$
\eta=\eta_{\text {exc }} \cdot \eta_{\text {rad }} \cdot \eta_{\text {ext }}
$$

$\eta_{\text {exc }}$ is the excitation efficiency. In the case of electrically driven devices, it represents the fraction of injected carriers that recombine in the active region. In the case of optically pumped devices, it accounts for the absorption efficiency of the phosphor. $\eta_{\text {rad }}$ is the radiative efficiency, often referred to as the internal quantum efficiency or QY. It represents the fraction of the excited or injected electron-hole pairs that recombine to emit a photon, and it is defined as the ratio of the radiative rate to the total recombination rate. The last factor in Equation (1) is the extraction efficiency $\eta_{\text {ext }}$, which is the fraction of blue/green/red light that escapes the device into free space. Because emitted light can be trapped within the device (via total internal reflection) and eventually absorbed, many light-emitting devices rely on the integration of light-extracting structures to increase $\eta_{\text {ext }}$. In addition to efficiency, other parameters defining the color of the emission must be assessed when developing new LEDs ${ }^{77}$.

\section{Metals and light emission}

Recently, various arrangements of metallic nanostructures have been proposed as a means of controlling light emission ${ }^{15-33}$. Research efforts in nanophotonics have mainly focused on using nanostructures to improve $\eta_{\text {rad }}$ via modification of the local density of optical states to which an emitter can decay ${ }^{15,78}$. For low-QY emitters (for example, $\mathrm{QY}<0.3$ ), this approach leads to a significantly brighter source. However, emitters with QY ca. 100\% are readily available for use in SSL. Therefore, enhancing the QY of poor emitters is not necessary for SSL applications. In fact, metallic nanostructures may reduce the overall efficiency of phosphor-based devices. Where metallic nanostructures benefit emission wavelength is in extracting specific emission colors in defined directions, thereby controlling the angular and spectral distributions of emitted light without diminishing significantly the device efficiency. Regarding $\eta_{\text {exc }}$, optical losses occurring at the excitation wavelength associated with the permittivity of metals are expected to be among the main limitations with regard to the overall efficiencies of plasmonic pcLEDs. A recent article addresses the role of metal absorption in the external QY of emitters coupled to arrays of plasmonic nanoparticles. It concludes that due to the reduced fraction of light absorbed by the metal nanoparticles control of the illumination conditions of the arrays can provide significant QY enhancement ${ }^{79}$.

SPPs are surface waves at the interface between a metal and a dielectric that are characterized by the manner in which they propagate along the surface while decaying evanescently away from it. These characteristics depend on the permittivities of the metal and the dielectric. SPPs result from the coherent oscillations, driven by the electromagnetic field, of the charge carriers in the metal. Emissions from sources in proximity to a metallic layer are strongly modified via the excitation of SPPs, which can be coupled to free space radiation by structuring the metal surface. A number of calculations describing the SPP modification of exciton decay rates have been reported ${ }^{40,80-83}$. As illustrated in Figure 2, SPP-coupled emission can be described 


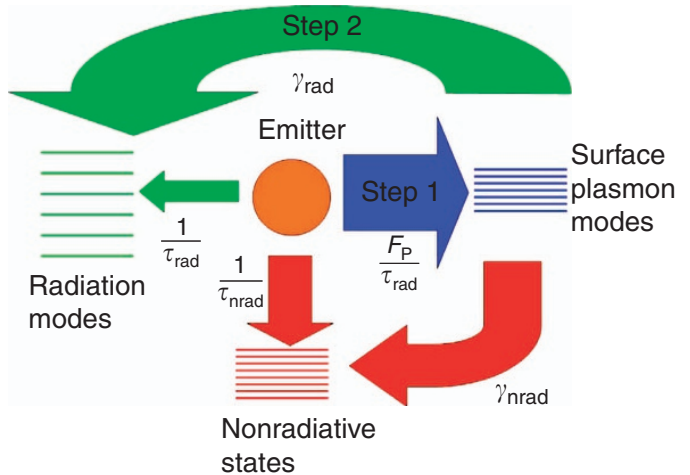

Figure 2 Sketch of the surface plasmon-coupled emission. It illustrates the coupling of an emitter into a SPP with the subsequent coupling of the energy of this plasmon mode into the radiation continuum. Reprinted with permission from Ref. 82. Copyright 2008 Optical Society of America.

according to a simple model based on coupling rates, in which the transfer of energy from the emitter to the plasmon modes occurs, followed by the radiative coupling of the plasmon modes into radiation. As discussed in the next section, this coupling has been used to improve the emission of LEDs. Complementary to metal layers are metal nanostructures that support localized optical resonances known as localized surface plasmon resonances (LSPRs). The LSPR frequency and linewidth depend on the geometry and size of the nanoparticles ${ }^{84}$, the permittivity of the metal and the surrounding medium. For emitters in close proximity to metallic nanoparticles, the fluorescence can be modified via processes occurring at the excitation and/or the emission wavelengths ${ }^{15,17}$, thereby involving all three terms in Equation (1). In addition, fluorescence enhancement depends strongly on the QY of the emitter ${ }^{81,85-90}$. Indeed, for high-QY emitters distributed over large areas, very little improvement of the QY can be obtained when using nanostructures that only modify the emissions of highly localized sources in their proximity ${ }^{80}$. Nevertheless, the directionality of the emission of a localized source coupled to a resonant metal nanoparticle can be modified as illustrated in Figure 3a.

Individual nanoantennas may be used to modify the emission of quantum wells, but they are not an option for influencing the emission of the much thicker phosphor layers. To circumvent this limitation, nanoparticles can be arranged in periodic arrays, such that their optical response is reinforced through coherent scattering. This leads to a collective plasmonic-photonic resonance first described by Carron et al. ${ }^{91}$. and Markel $^{92}$ in the context of surface-enhanced Raman scattering. Schatz and co-workers later revived interest in this collective resonance phenomenon via a series of theoretical papers that demonstrate the emergence of very sharp resonances (ca. $1-\mathrm{meV}$ linewidth) in the extinction spectra of arrays composed of metal nanoparticles ${ }^{93-103}$. Such sharp resonances signify the extremely low radiation losses that collective resonances feature. In-plane scattering by the nanoparticles and phase accumulation of these scattered fields govern the optical response of the array ${ }^{104}$. Periodic arrays in homogeneous dielectrics are characterized by narrow resonances called surface lattice resonances (SLRs). Interestingly, SLRs with linewidths as narrow as ca. $8 \mathrm{meV}$ have been obtained experimentally using carefully designed periodic arrangements of gold nanorods ${ }^{103}$. These comprise the sharpest plasmonic resonances ever reported, reaching values close to those theoretically predicted by Schatz, which are the limit of meaningful expectations for lighting applications. In addition, if an optical waveguide is placed near the array, waveguide-plasmon a
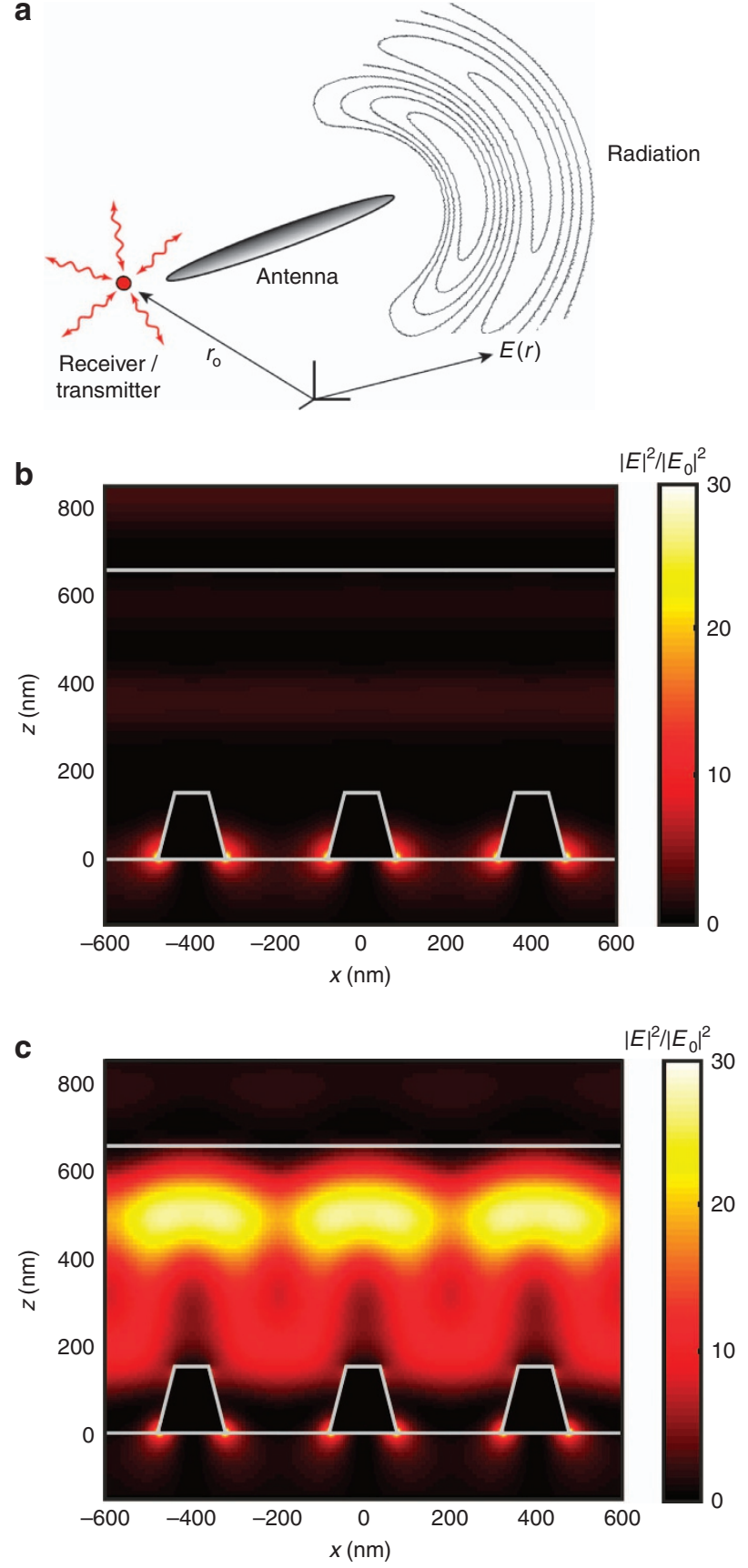

Figure 3 (a) Sketch of the interaction between a light emitter and an optical antenna. Reprinted with permission from Ref. 25. Copyright 2009 Optical Society of America. Side view of the simulated spatial field distribution in three unit cells of the array at a frequency corresponding to (b) a localized surface plasmon resonance and (c) a collective resonance ${ }^{33}$.

polaritons can be excited ${ }^{105-108}$. In the case of SLRs, the nanoparticles interact with Rayleigh anomalies, which are the onset of diffraction orders, that is, the frequency at which diffraction orders propagate in the plane of the array. This in-plane diffraction enhances the radiative coupling between the individual nanoparticles, giving rise to a collective resonance. For waveguide-plasmon polaritons, this radiative coupling between nanoparticles is enhanced by guided modes in highrefractive index layers ${ }^{108}$. In the particular case of light emission, 
emitters in the proximity of an array can decay into either of these collective resonances and consequently radiate into free space with a spectrum and directionality determined by the dispersion of these collective modes ${ }^{107,108}$.

Figure $3 \mathrm{~b}$ and $3 \mathrm{c}$ illustrates the electromagnetic field enhancements characteristic of two distinct resonances in a periodic array of metal nanoparticles. Figure $3 \mathrm{~b}$ represents a cross section at the LSPR condition. Associated with the LSPRs is a large electromagnetic field enhancement that is highly localized to the individual particles, as seen near the base of the nanostructures in Figure 3b. In contrast, Figure $3 \mathrm{c}$ shows a collective resonance where the field is enhanced over a much larger volume. These extended field enhancements enable circumvention of the limitations regarding critical placement of the emitter relative to the metal nanoparticles ${ }^{109}$. Moreover, the emission from such an extended state can be highly directional ${ }^{110,111}$, as shown below. These are the key advantages of using collective resonances for modifying the emission of phosphor layers ${ }^{33}$. The metallic nanoparticle array behaves as a phased array of optical antennas, with the relative phases between antennas determined by their resonant responses and separations. The radiation patterns of subwavelength sources are modified by coherent scattering within the periodically spaced metal nanoparticles ${ }^{112}$.

It is noteworthy that although this review focuses on the impact of metallic nanostructures on the performance of light-emitting devices, metallic and dielectric nanostructures represent complementary approaches to modifying the emission properties of light sources. Several reviews that discuss modification of the emission characteristics of LEDs using dielectric materials have been reported in the past few years ${ }^{113-115}$. In particular, wavelength-sized dielectric structures have demonstrated improved light extraction ${ }^{116-118}$, and accurate control of radiation patterns ${ }^{119}$ and polarizations ${ }^{120}$. A recent report demonstrated that the effects of periodic arrays of Si nanoparticles on the performance of thin layers of emitters are similar to those of their plasmonic counterparts ${ }^{121}$. Choosing between metals and dielectrics for enhancement of light emission will depend on fabrication constraints and/or the limitations associated with the particular goal.

\section{PLASMONIC-BASED LIGHT EMISSION ENHANCEMENT}

\section{Plasmon-enhanced emission from quantum wells}

In this section we focus on the different nanophotonics-based approaches that have been used to improve the efficiencies of inorganic semiconductor LEDs. Inorganic blue LEDs that are based on InGaN/GaN multi-quantum-well heterostructures are currently used in advanced architectures to obtain white-light emission. However, light generated in the active region of the multi-quantum-well structure can be reflected at the interfaces and trapped in the layered structure before it reaches the phosphor. To remedy this and maximize light extraction, metallic surfaces and nanostructures have been used.

The metallic thin films used with SPPs have been applied directly to LEDs to enhance the spontaneous emission rate of excitons in quantum wells and, therefore, the $\mathrm{QY}^{37,39-41,122-124}$. The process can be explained as follows. Electron-hole pairs are injected in the active region of the LED. When a metal layer is grown at a distance smaller than the evanescent decay length of the SPPs, the electron-hole pairs recombine, giving their energy to the SPPs. Thus, the metal provides additional states for exciton recombination ${ }^{125}$. This enhanced density of states for exciton recombination can significantly increase the recombination rate. Because SPPs are evanescent surface waves, they cannot radiate to free space. The metallic surface can be made rough to efficiently couple SPPs to free space radiation and enhance the a

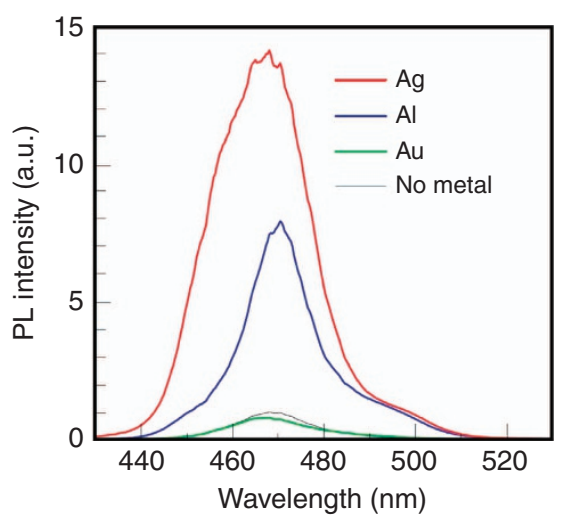

b

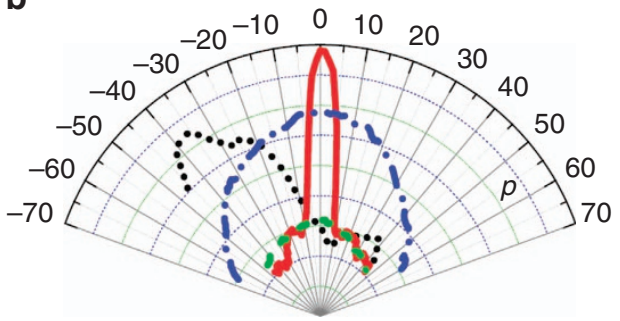

Figure 4 (a) Photoluminescence spectra of blue LEDs coated with $\mathrm{Ag}, \mathrm{Al}$ and $\mathrm{Au}$. The enhanced emission of the coated LEDs is due to the outcoupling of SPPs with the roughness of the metal to far-field radiation. Reprinted by permission from Macmillan Publishers Ltd ${ }^{122}$, copyright 2004. (b) Far-field radiation patterns of blue LEDs that feature a one-dimensional grating (red solid curve) and triangular nanoantennas (black dotted curve), compared with those of the bare LED sample (blue dashed-dotted curve) and a flat metallic coating (green dashed curve). Reprinted with permission from Ref. 126. Copyright 2013, AIP Publishing LLC.

emission intensity. To illustrate this effect, Figure 4a shows the photoluminescence intensity spectrum for different metal layers deposited over the blue LED at a distance of $10 \mathrm{~nm}$. Enhancement of the visible light emission originates from a combined higher recombination rate and a higher quantum-well extraction efficiency enabled by the nanometer-sized roughness in the metal layer. Although such random textures result in improved extraction efficiencies, they provide little control over the directionality of the emitted light, which typically displays a Lambertian profile ${ }^{127}$.

Accurate control over the angular distribution of the emission can be achieved using metallic nanostructures, which are directly fabricated, with predetermined geometries and dimensions, on the emissive semiconductor surface ${ }^{80,128-130}$. Aperiodic designs may also be used to avoid undesirable angular and/or spectral dependencies ${ }^{131}$. Unidirectional beaming of the LED emission has been recently demonstrated using a periodic array of optical antennas with specifically designed geometries ${ }^{126}$. Figure $4 \mathrm{~b}$ shows the far-field radiation pattern of such a nanostructured blue LED. In the absence of the metallic nanostructure, a broad Lambertian emission is observed (blue dashed dotted curve). The silver flat film causes a substantial reduction in the intensity of the emitted light for both polarizations (green dashed curve), because no mechanism is provided to scatter the excited SPPs into radiation. In contrast, in the direction of the maximum intensity for one polarization, the output intensity of the LED with metallic nanostructures (black dotted and red solid curves) is enhanced compared with that of the flat sample. This polarization dependence can be attributed to the asymmetric shape of the nanostructures. Emission enhancements with a preferential light polarization can be 
beneficial for applications where light impinges upon smooth surfaces at nearly grazing angles, for example, automotive lighting. In these cases, it may be desirable to selectively enhance the emission obtained for one polarization only, because the other polarization may lead to unwanted effects, such as glare from incoming drivers.

\section{Plasmon-enhanced emission from phosphor layers}

We now turn our attention to emission modifications obtained using extended emitting layers. Phosphor layers are typically much thicker than quantum wells. The potential of metallic nanoparticle arrays to modify the Lambertian emission from such thick luminescent layers has been demonstrated in several reports ${ }^{24-33,42,108,132,133}$. This section provides few examples of how these arrays modify the emissions of layers of emitters. In contrast to LSPRs, which are usually characterized by their broadband responses and weak angular dependence, collective plasmonic-photonic resonances in antenna arrays can be spectrally very narrow and exhibit strong angular dependence.

Vecchi et al. ${ }^{24}$ first demonstrated the use of collective plasmonic resonances to modify the emission of luminescence layers, using nearinfrared-emitting dye molecules and arrays of gold nanoparticles. Lozano et al. ${ }^{33}$ proposed that these systems be applied to SSL by using highly efficient visible-light-emitting dye molecules as extended phosphor layers and arrays of aluminum nanoparticles. In this work, a sample such as the one shown in Figure 5 a was used to enhance the emission of a 700-nm-thick phosphor layer consisting of a high-QY and a photo-stable dye by more than a factor of 60 at certain wavelengths and in defined directions. This enhancement is illustrated in Figure $5 \mathrm{~b}$ and $5 \mathrm{c}$ for normal incidence, where the extinction and photoluminescence enhancement (PLE) spectra are displayed, respectively. The latter is given by the ratio of the emission from the dye layer with and without the nanoantenna array. The enhanced directional emission can be described as follows. The photo-excited dye molecules relax, exciting collective resonances in the particle array. The periodic structure of the array is responsible for the directional outcoupling of the emission in defined directions. The narrow linewidths of the emission associated with the collective modes are a direct consequence of the enhancement of the spatial coherence of the emission due to the coherent scattering by the nanoantennas. The 60 -factor fluorescence enhancement of Ref. 33 can be attributed to a ca. 6-fold enhancement of the excitation efficiency $\left(\eta_{\text {exc }}\right)$ and a ca. 10-fold enhancement of the extraction efficiency $\left(\eta_{\text {ext }}\right)$. Moreover, based on time-resolved measurements, only a very small modification of the QY was determined (ca. 15\% degradation at most). A visualization of this enhanced emission is shown in Figure $5 d$, which is a photograph of the emission of a phosphor layer on top of a nanoantenna array (right side). For direct comparison, a reference sample consisting of the phosphor layer with the same thickness but without the antenna array is also shown (left side).

The directionality of this emission enhancement can be controlled depending on the application. Figure $5 \mathrm{e}-5 \mathrm{~h}$ highlights recent results demonstrating tailored enhanced directional emission in narrow angular ranges for red light $(\lambda=620 \mathrm{~nm})$ with hexagonal arrays of nanoantennas ${ }^{42}$. This spectral region is of particular interest with regard to achieving warm white light in SSL applications. High symmetry lattices, such as the hexagonal array, facilitate a more homogeneous distribution of the emission over the azimuthal angle, as shown in the photoluminescence intensity polar plot measurements displayed in Figure 5e-5h. Manipulating the separation distance between aluminum particles enables accurate control over the directionality of the red emitted light in pcLEDs ${ }^{42}$.

The inclusion of metallic nanoparticles minimizes the need for optical components in LEDs, such as parabolic mirrors or condenser lenses that are used for beaming the emission. These optical elements are often bulky, increasing the total size of the LEDs and limiting their a

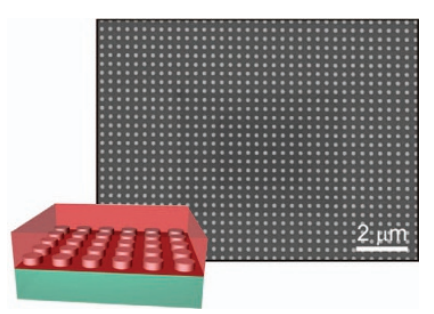

b

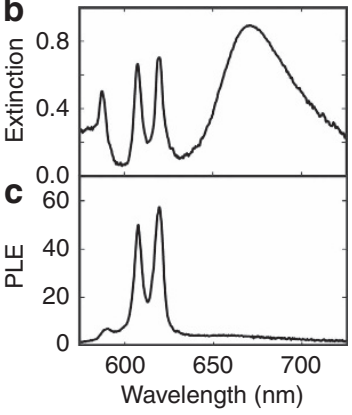

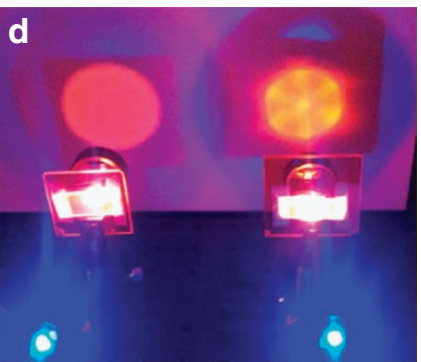

PL intensity
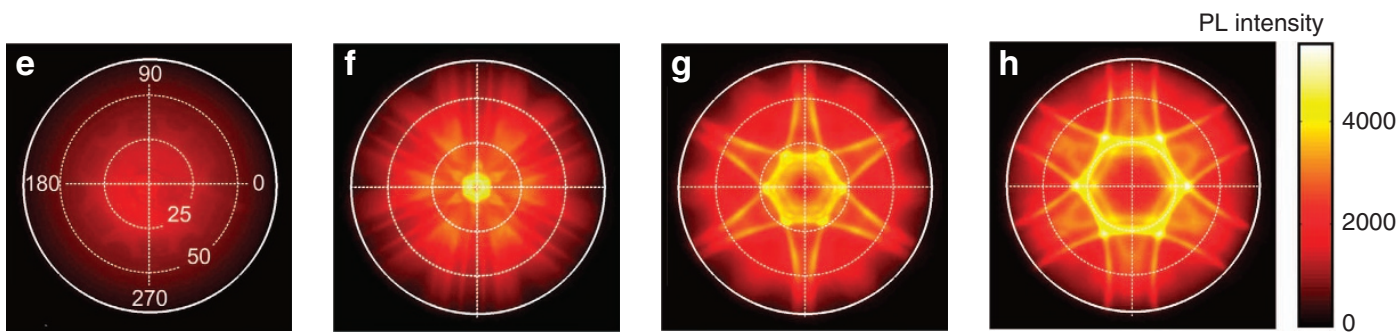

Figure 5 (a) Scanning electron micrograph of a square array of aluminum nanoparticles. The inset is a sketch of a metal nanoparticle array pcLED, where the phosphor layer is represented by the transparent red layer. (b) Extinction and (c) PLE of an aluminum nanoparticle array covered by a thin phosphor layer. Reprinted with permission from Macmillan Publishers Ltd ${ }^{33}$, copyright 2013. (d) Photograph of the emission of a standard pcLED (left) and a pcLED that exhibits enhanced emission due to the integration of a hexagonal array of metal nanoparticles (right). (e) Fourier image of the unpolarized red emission (610$620 \mathrm{~nm}$ ) of an unstructured pcLED. (f-h) Fourier images of the unpolarized red emission of a similar pcLED that features a hexagonal array of Al nanoparticles with lattice constants (f) $475 \mathrm{~nm}$, (g) $425 \mathrm{~nm}$ and (h) $375 \mathrm{~nm}$. Reproduced from Ref. 42 with permission from The Royal Society of Chemistry. 
integration. Therefore, the performance of metallic nanoparticle arrays in SSL applications must be assessed in terms of overall system efficiency with and without the presence of the metallic nanoparticle arrays. From a device perspective, the enhancement of phosphor-layer emissions enabled by the use of nanoparticles must not only be compared with emissions obtained from the same layer when no nanoparticles are used. We must also compare the results obtained with the same phosphor layer under conditions in which the usual secondary optical elements are in place.

An additional advantage of nanoantenna-enhanced emission is that it also reduces the phosphor-layer thickness, which is important with regard to heat dissipation. Heat reduces emission efficiency, limiting the performance of LEDs. So far, it has not been easy to use thin layers in pcLEDs owing to their low blue absorption; the conversion efficiencies of these layers have not been sufficient to generate the desired emission spectrum. Therefore, the combination of metallic arrays and new phosphors, such as dye molecules or quantum dots, enables the use of layers that are much thinner than standard YAG:Ce pallets, resulting in improved heat management and high extraction efficiencies.

\section{OUTLOOK AND CONCLUSIONS}

LEDs constitute a new technology that is currently driving substantial changes in the way artificial light is generated. Metallic nanostructures enable strong light-matter interactions that facilitate unprecedented improvements in the emission intensities, colors and directionalities of light sources positioned nearby. This review presents and discusses new methods for enhancing the efficiency of LEDs using metals structured on the nanometer scale. We have provided a general overview of state-of-the-art LED lighting, discussing the main requirements of both quantum wells and phosphors for efficient generation of white light. We also discuss the main challenges facing researchers in this regard and the potential of plasmonics to overcome them. In what follows, we highlight a few recent findings in plasmonics that may lead to new illumination solutions and provide perspectives for future progress.

Several applications, for example, screen or automotive lighting, require light to be directed in only one direction. For planar structures, such as shallow nanoantenna arrays, light beaming into small angles is enhanced with roughly equal strengths in the forward and backward directions. The light emitted backward must be recycled using secondary optics, resulting in losses. To address this issue, the forward-backward light emission symmetry of planar structures can be broken by integrating an array of nanostructures with a pyramidal shape into the fluorescent layer ${ }^{134}$. Figure 6 shows the PLE spectrum of an aluminum nanopyramid array. Notice that the PLE differs toward the top (black curve) and bottom (red curve) of the nanopyramids. At the LSPR wavelength $(\sim 650 \mathrm{~nm})$, the nanopyramid array beams more light toward the bottom of the pyramids. The opposite occurs at the SLR wavelength $(\sim 585 \mathrm{~nm})$. These effects are due to the enhanced magnetoelectric response of the nanopyramid array (magnetic dipole moments are excited via the electric field of light), which originates from the pyramidal shape and height of the nanostructures ${ }^{134}$. Future research should further investigate these phenomena in order to increase emission asymmetry and maximize the fraction of the emitted intensity that can be efficiently used in SSL.

Beyond passive spectral and directional control of light emission, a long-standing goal in nanophotonics is to actively control emitted light properties by means of an external tuning parameter. This can be achieved by incorporating materials with optical properties that

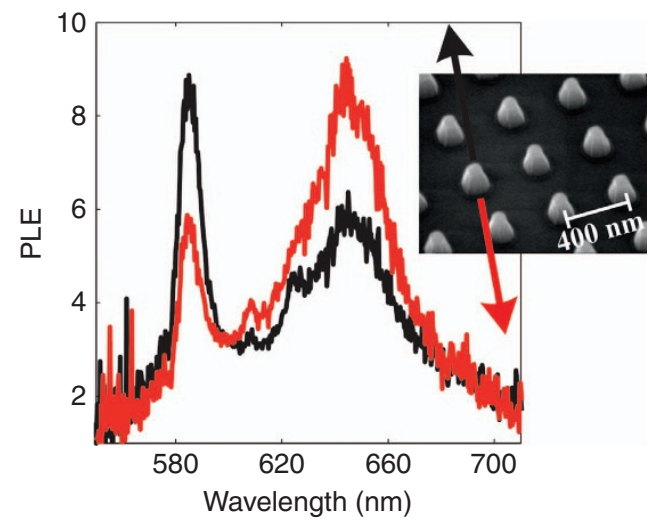

Figure 6 PLE toward the top (black curve) and bottom (red curve) of the pyramids. The inset displays a scanning electron micrograph of the fabricated structures before the deposition of the luminescent layer. Reprinted with permission from Ref. 134. Copyright 2014, American Physical Society.

depend on applied voltages, heat, strain or illumination profiles ${ }^{135-138}$. Liquid crystals are particularly interesting in this regard. Their tunable orientations have been used to actively control the properties of LSPRs and SPPs ${ }^{139-145}$. Active light emission from antenna arrays has recently been demonstrated with liquid crystals $^{146}$. Further research in this regard is sure to attain active control of the color, direction, polarization and intensity of emission from pcLEDs.

Thus far, the examples of spontaneous emission modifications of light emitters coupled to plasmonic resonances provided in this review involve the so-called weak coupling regime. Recently, strong coupling has attracted more attention. Strong coupling is characterized by an emitter-resonator energy exchange rate that exceeds all loss rates. The energy exchange rate between an ensemble of emitters, such as those found in macroscopic light-emitting devices, and an optical mode depends on the concentrations of the emitters. When the collective oscillator strength of the ensemble of emitters becomes comparable to that of the optical mode, that is, at high densities of emitters, mixed light-matter states known as polaritons can be created. Strong coupling between emitters and SPPs has been investigated with regard to propagating modes in flat ${ }^{147-149}$ and perforated ${ }^{150-152}$ metallic layers, as well as creating localized modes in nanostructures ${ }^{153-156}$. Recently, strong coupling between SLRs and emitters has also been observed $^{157-159}$. Törma and Barnes ${ }^{160}$ have recently published a review article in this emerging field. Although the physics of strongly coupled plasmon-emitter systems is very rich, and the prospect of strongly interacting emitters is exciting, the potential of these systems for use in light-emitting devices has rarely been discussed. One of the challenges in this regard is related to the poor QY that phosphor layers with high densities of organic molecules display. Although it is required to access the strong coupling regime, a high molecular density degrades the QY of the ensemble via an effect known as 'concentration quenching'161. Therefore, challenges remain with regard to improving high-QY light-emitting devices via strong emitter-plasmon coupling.

Laser diodes are being considered as an alternative to LEDs for future SSL applications to circumvent the strong reduction of $\eta_{\text {rad }}$ that blue LEDs experience at high current densities. This issue, called the efficiency droop ${ }^{162-164}$, is one of the biggest challenges with regard to utilizing blue-light-emitting materials and devices for SSL ${ }^{165}$. Photonic 
a

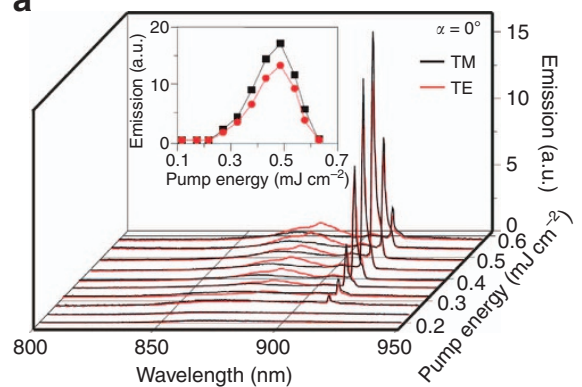

b

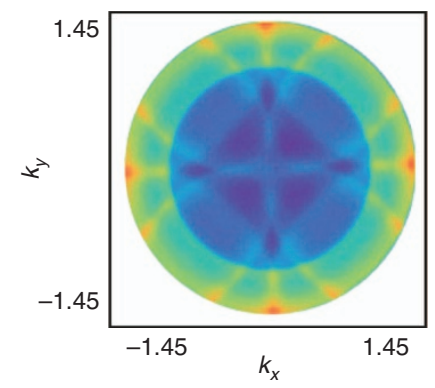

d

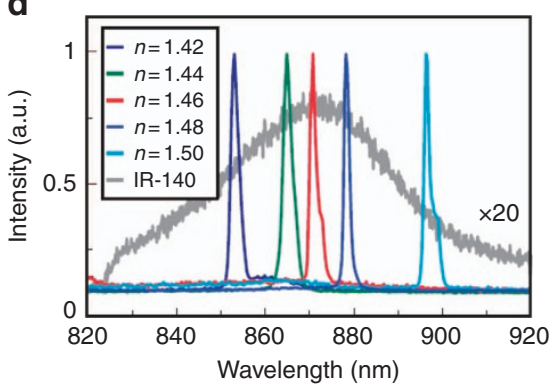

C

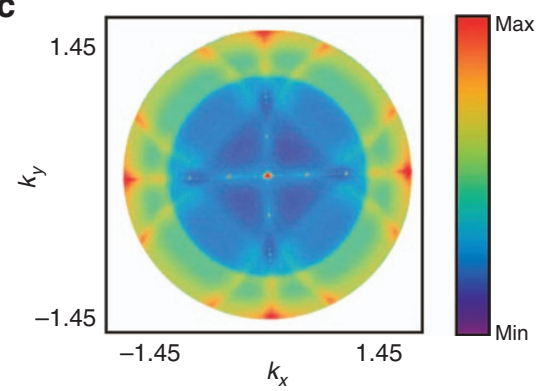

Figure 7 (a) Emission spectra as a function of the pump power for a plasmonic array laser. The inset shows the output emission intensity as a function of the input pump pulse energy. Reprinted with permission from Macmillan Publishers Ltd ${ }^{32}$, copyright 2013. (b,c) Fourier images below and just above threshold for an array with a lattice constant of $380 \mathrm{~nm}$. Reprinted with permission from Ref. 174. Copyright 2014, American Physical Society. (d) Lasing emission from Au arrays embedded in different refractive index environments. Reprinted from Ref. 175, Macmillan Publishers Ltd, licensed under Creative Commons Attribution 4.0.

band-edge lasers have been extensively investigated during the past two decades ${ }^{16-169}$. Plasmonic nanolasers provide faster dynamics and offer the opportunity to realize ultracompact devices at the expense of beam directionality ${ }^{170}$. Diffractive plasmon lattices overcome this limitation ${ }^{32,171-175}$. Figure 7 a displays the lasing threshold curve for an Au particle array combined with a dye-doped polymer acting as a gain medium ${ }^{32}$. Figure $7 b$ and $7 c$ shows the Fourier images of the emissions below and above threshold for an array made of $\mathrm{Ag}$ nanoparticles, respectively, illustrating the highly directional nature of the lasing mode. Moreover, it has been demonstrated that the lasing modes in these sorts of arrays can be modified with liquids of different indexes of refraction, as illustrated in Figure $7 \mathrm{~d}^{175}$.

In conclusion, we have reviewed different metallic nanostructures that provide new possibilities for efficient light management in the next generation of LED devices. A central goal of SSL technology is to replace all incandescent light bulbs worldwide. Consequently, efficiency and cost are currently driving the market. Solutions implying an increase in the overall cost of the lighting device are currently not viable for general lighting purposes. However, this situation will change if the market becomes design- or performance-driven or once the first generation of LED luminaires becomes obsolete. Such a paradigm shift will open the door to the integration of cost-effective nanophotonic structures, which will in turn enable realization of more compact devices with fine control over the intensity, directionality and color quality of the resulting emitted light. Several parameters are typically used to assess the performance of LED-based lighting, that is, quantum efficiency, luminous efficacy, correlated color temperature and color-rendering index (CRI), and maximizing all of them simultaneously is unfeasible. Indeed, there exists a clear trade-off between luminous efficacy and light quality. White-light sources require an emission spectrum that extends throughout the visible range, thereby significantly reducing their maximum luminous efficacy (which is attained for a light source that converts 100\% of its electrical power into green radiation, to which the human eye is most sensitive). A wide variety of applications can benefit from LED devices that integrate different emission characteristics. In particular, for general illumination applications, the light source must show a high correlated color temperature as well as a CRI of at least 90 and a uniform colorover-angle. Even higher CRI values, that is, toward 100, are required in museums or in retail businesses. In contrast, highly directional sources are sought for automotive lighting, where the compactness and the freedom to design the esthetics of the light source are more important than the efficiency or quality of the emitted light. Finally, directional narrowband sources are needed in the light guides used in screens. Metallic nanostructures offer new opportunities for tailoring the emission spectra of luminescent materials that compose emitting devices as well as associated angular dependence. Such fine emission control, as illustrated by the different approaches highlighted in this review, should certainly improve the performances of devices designed to for the aforementioned applications. The overall benefit of nanostructures is, in many cases, still limited due to increased complexities in device fabrication. Nonetheless, tuning light emission in LEDs continues to be a key challenge of fundamentally interest and technological relevance.

\section{ACKNOWLEDGEMENTS}

This research was financially supported by the Nederlandse Organisatie voor Wetenschappelijk Onderzoek (NWO) through the project LEDMAP of the Technology Foundation STW and through the Industrial Partnership Program Nanophotonics for Solid State Lighting between Philips and the Foundation for Fundamental Research on Matter FOM. It is also supported by NanoNextNL of the Government of the Netherlands and 130 partners. 
1 Schubert EF, Kim JK. Solid-state light sources getting smart. Science 2005; 308: 1274-1278.

2 Tsao JY, Crawford MH, Coltrin ME, Fisher AJ, Koleske DD et al. Toward smart and ultra-efficient solid-state lighting. Adv Opt Mater 2014; 2: 809-836.

3 Round HJ. A note on carborundum. Electr World 1907; 49: 308.

4 Losev OV. Luminous carborundum detector and detection with crystals. Telegr Telef Prov 1927; 44: 485-494.

5 Wolff GA, Hebert RA, Broder JD. Electroluminescence of GaP. Phys Rev 1955; 100 : 1144-1145.

6 Braunstein R. Radiative transitions in semiconductors. Phys Rev 1955; 99 1892-1893.

7 Holonyak Jr N, Bevacqua SF. Coherent (visible) light emission from Ga( $\left.\mathrm{As}_{1-x} \mathrm{P}_{x}\right)$ junctions. Appl Phys Lett 1962; 1: 82-83.

8 Pankove Jl. Tunneling-assisted photon emission in gallium arsenide pn junctions. Phys Rev Lett 1962; 9: 283-285.

9 Itoh K, Kawamoto T, Amano H, Hiramatsu K, Akasaki I. Metalorganic vapor phase epitaxial growth and properties of $\mathrm{GaN} / \mathrm{Al}_{0.1} \mathrm{Gaa}_{0.9} \mathrm{~N}$ layered structures. Jpn J App/ Phys 1991; 30: 1924

10 Nakamura S, Senoh M, Mukai T. p-GaN/N-InGaN/N-GaN double-heterostructure bluelight-emitting diodes. Jpn J Appl Phys 1993; 32: L8.

11 Nakamura S, Mukai T, Senoh M. Candela-class high-brightness InGaN/AIGaN doubleheterostructure blue-light-emitting diodes. Appl Phys Lett 1994; 64: 1687-1689.

12 Krames MR, Shchekin OB, Mueller-Mach R, Mueller GO, Zhou L et al. Status and future of high-power light-emitting diodes for solid-state lighting. J Disp Technol 2007; 3: 160-175.

13 Koenderink AF, Alù $\mathrm{A}$, Polman A. Nanophotonics: shrinking light-based technology. Science 2015; 348: 516-521.

14 Barnes WL, Dereux A, Ebbesen TW. Surface plasmon subwavelength optics. Nature 2003; 424: 824-830.

15 Biteen JS, Pacifici D, Lewis NS, Atwater HA. Enhanced radiative emission rate and quantum efficiency in coupled silicon nanocrystal-nanostructured gold emitters. Nano Lett 2005; 5: 1768-1773.

16 Anger P, Bharadwaj P, Novotny L. Enhancement and quenching of single-molecule fluorescence. Phys Rev Lett 2006; 96: 113002.

17 Kühn S, Håkanson U, Rogobete L, Sandoghdar V. Enhancement of single-molecule fluorescence using a gold nanoparticle as an optical nanoantenna. Phys Rev Lett 2006; 97: 017402.

18 Pillai S, Catchpole KR, Trupke T, Zhang G, Zhao J et al. Enhanced emission from Sibased light-emitting diodes using surface plasmons. Appl Phys Lett 2006; 88: 161102.

19 Muskens OL, Giannini V, Sánchez-Gil JA, Rivas JG. Strong enhancement of the radiative decay rate of emitters by single plasmonic nanoantennas. Nano Lett 2007; 7: 2871-2875.

20 Biteen JS, Sweatlock LA, Mertens H, Lewis NS, Polman A et al. Plasmon-enhanced photoluminescence of silicon quantum dots: simulation and experiment. J Phys Chem C 2007; 111: 13372-13377.

21 Iwase H, Englund D, Vučković J. Spontaneous emission control in high-extraction efficiency plasmonic crystals. Opt Express 2008; 16: 426-434.

22 Ringler M, Schwemer A, Wunderlich M, Nichtl A, Kürzinger $K$ et al. Shaping emission spectra of fluorescent molecules with single plasmonic nanoresonators. Phys Rev Lett 2008; 100: 203002.

23 Kinkhabwala A, Yu Z F, Fan S H, Avlasevich Y, Müllen K et al. Large single-molecule fluorescence enhancements produced by a bowtie nanoantenna. Nat Photon 2009; 3: 654-657.

24 Vecchi G, Giannini V, Rivas JG. Shaping the fluorescent emission by lattice resonances in plasmonic crystals of nanoantennas. Phys Rev Lett 2009; 102: 146807.

25 Bharadwaj P, Deutsch B, Novotny L. Optical antennas. Adv Opt Photonics 2009; 1: 438-483.

26 Walters RJ, van Loon RVA, Brunets I, Schmitz J, Polman A. A silicon-based electrical source of surface plasmon polaritons. Nat Mater 2010; 9: 21-25.

27 Schuller JA, Barnard ES, Cai W S, Jun YC, White JS et al. Plasmonics for extreme light concentration and manipulation. Nat Mater 2010; 9: 193-204.

28 Curto AG, Volpe G, Taminiau TH, Kreuzer MP, Quidant R et al. Unidirectional emission of a quantum dot coupled to a nanoantenna. Science 2010; 329: 930-933.

29 Aouani H, Mahboub O, Bonod N, Devaux E, Popov E et al. Bright unidirectional fluorescence emission of molecules in a nanoaperture with plasmonic corrugations. Nano Lett 2011; 11: 637-644.

30 Aouani H, Mahboub O, Devaux E, Rigneault H, Ebbesen TW et al. Plasmonic antennas for directional sorting of fluorescence emission. Nano Lett 2011; 11: 2400-2406.

31 Rodriguez SRK, Lozano G, Verschuuren MA, Gomes R, Lambert K et al. Quantum rod emission coupled to plasmonic lattice resonances: a collective directional source of polarized light. App/ Phys Lett 2012; 100: 111103.

32 Zhou W, Dridi M, Suh JY, Kim CH, Co DT et al. Lasing action in strongly coupled plasmonic nanocavity arrays. Nat Nanotechnol 2013; 8: 506-511.

33 Lozano G, Louwers DJ, Rodriguez SRK, Murai S, Jansen OTA et al. Plasmonics for solid-state lighting: enhanced excitation and directional emission of highly efficient light sources. Light Sci App/ 2013; 2: e66.

34 Langguth L, Punj D, Wenger J, Koenderink AF. Plasmonic band structure controls single-molecule fluorescence. ACS Nano 2013; 7: 8840-8848.

35 Greffet JJ. Nanoantennas for light emission. Science 2005; 308: 1561-1563.

36 Mühlschlegel P, Eisler HJ, Martin OJF, Hecht B, Pohl DW. Resonant optical antennas. Science 2005; 308: 1607-1609.
37 Gontijo I, Boroditsky M, Yablonovitch E, Keller S, Mishra UK et al. Coupling of InGaN quantum-well photoluminescence to silver surface plasmons. Phys Rev B 1999; 60: 11564

38 Eggleston MS, Messer K, Zhan L, Yablonovitch E, Wu MC. Optical antenna enhanced spontaneous emission. Proc Natl Acad Sci USA 2015; 112: 1704-1709.

39 Hecker NE, Höpfel RA, Sawaki N. Enhanced light emission from a single quantum well located near a metal coated surface. Physica E 1998; 2: 98-101.

40 Vuckovic J, Loncar M, Scherer A. Surface plasmon enhanced light-emitting diode. EEE J Quantum Electron 2000; 36: 1131-1144.

41 Neogi A, Lee CW, Everitt HO, Kuroda T, Tackeuchi A et al. Enhancement of spontaneous recombination rate in a quantum well by resonant surface plasmon coupling. Phys Rev B 2002; 66: 153305.

42 Lozano G, Grzela G, Verschuuren MA, Ramezani M, Rivas JG. Tailor-made directional emission in nanoimprinted plasmonic-based light-emitting devices. Nanoscale 2014; 2014: 9223-9229.

43 Kido J, Kimura M, Nagai K. Multilayer white light-emitting organic electroluminescent device. Science 1995; 267: 1332-1334.

44 Sheats JR, Antoniadis H, Hueschen M, Leonard W, Miller J et al. Organic electroluminescent devices. Science 1996; 273: 884-888.

45 D'Andrade BW, Forrest SR. White organic light-emitting devices for solid-state lighting. Adv Mater 2004; 16: 1585-1595.

46 So F, Kindo J, Burrows P. Organic light-emitting devices for solid-state lighting. MRS Bull 2008; 33: 663-669.

47 Reineke S, Lindner F, Schwartz G, Seidler N, Walzer K et al. White organic lightemitting diodes with fluorescent tube efficiency. Nature 2009; 459: 234-238.

48 Tyan YS. Organic light-emitting-diode lighting overview. J Photon Energy 2011; 1: 011009.

49 Gather MC, Köhnen A, Meerholz K. White organic light-emitting diodes. Adv Mater 2011; 23: 233-248.

50 Reineke S, Thomschke M, Lüssem B, Leo K. White organic light-emitting diodes: status and perspective. Rev Mod Phys 2013; 85: 1245-1293.

51 Pei QB, Yu G, Zhang C, Yang Y, Heeger AJ. Polymer light-emitting electrochemical cells. Science 1995; 269: 1086-1088.

52 Meier SB, Tordera D, Pertegás A, Roldán-Carmona C, Ortí E et al. Light-emitting electrochemical cells: recent progress and future prospects. Mater Today 2014; 17: 217-223.

53 Pimputkar S, Speck JS, DenBaars SP, Nakamura S. Prospects for LED lighting. Nat Phtoton 2009; 3: 180-182.

54 Žukauskas A, Shur MS, Gaska R. Introduction to Solid-State Lighting. New York: Wiley. 2002

55 Steigerwald DA, Bhat JC, Collins D, Fletcher RM, Holcomb MO et al. Illumination with solid state lighting technology. IEEE J Sel Topics Quantum Electron 2002; 8: 310-320

56 Shur MS, Žukauskas A. Solid-state lighting: toward superior illumination. Proc IEEE 2005; 93: 1691-1703

57 Hong YJ, Lee $\mathrm{CH}$, Yoon A, Kim M, Seong $\mathrm{HK}$ et al. Visible-color-tunable lightemitting diodes. Adv Mater 2011; 23: 3284-3288.

58 Qian F, Gradečak S, Li Y, Wen CY, Lieber CM. Core/multishell nanowire heterostructures as multicolor, high-efficiency light-emitting diodes. Nano Lett 2005; 5: 2287-2291.

59 Funato M, Hayashi K, Ueda M, Kawakami Y, Narukawa Y et al. Emission color tunable light-emitting diodes composed of InGaN multifacet quantum wells. App/ Phys Lett 2008; 93: 021126.

60 Limbach F, Hauswald C, Lähnemann J, Wölz M, Brandt O et al. Current path in light emitting diodes based on nanowire ensembles. Nanotechnology 2012; 23: 465301.

61 Li SF, Waag A. GaN based nanorods for solid state lighting. J Appl Phys 2012; 111 : 071101.

62 Bengoechea-Encabo A, Albert S, Lopez-Romero D, Lefebvre P, Barbagini F et al. Light-emitting-diodes based on ordered InGaN nanocolumns emitting in the blue, green and yellow spectral range. Nanotechnology 2014; 25: 435203.

63 Höppe HA. Recent developments in the field of inorganic phosphors. Angew Chem Int Ed Eng/ 2009; 48: 3572-3582.

64 Smet PF, Parmentier AB, Poelman D. Selecting conversion phosphors for white lightemitting diodes. J Electrochem Soc 2011; 158: R37-R54.

65 Lin CC, Liu RS. Advances in phosphors for light-emitting diodes. J Phys Chem Lett 2011; 2: 1268-1277.

66 Demir HV, Nizamoglu S, Erdem T, Mutlugun E, Gaponik N et al. Quantum dot integrated LEDs using photonic and excitonic color conversion. Nano Today 2011; 6 : 632-647.

67 Dohnalová K, Poddubny AN, Prokofiev AA, de Boer WDAM, Umesh CP et al. Surface brightens up Si quantum dots: direct bandgap-like size-tunable emission. Light Sci Appl 2013; 2: e47.

68 Li XF, Budai JD, Liu F, Howe JY, Zhang JH et al. New yellow $\mathrm{Ba}_{0.93} \mathrm{Eu}_{0.07} \mathrm{Al}_{2} \mathrm{O}_{4}$ phosphor for warm-white light-emitting diodes through single-emitting-center conversion. Light Sci App/ 2013; 2: e50.

69 Zhang R, Lin H, Yu YL, Chen DQ, Xu J et al. A new-generation color converter for highpower white LED: transparent $\mathrm{Ce}^{3+}$ : YAG phosphor-in-glass. Laser Photon Rev 2014; 8: 158-164.

70 Pust P, Weiler V, Hecht C, Tücks A, Wochnik AS et al. Narrow-band red-emitting Sr $\left[\mathrm{LiAl}_{3} \mathrm{~N}_{4}\right]: \mathrm{Eu}^{2+}$ as a next-generation LED-phosphor material. Nat Mater 2014; 13 : 891-896.

71 Pust P, Schmidt PJ, Schnick W. A revolution in lighting. Nat Mater 2015; 14: $454-458$. 
72 Zhu HM, Lin CC, Luo WQ, Shu ST, Liu ZG et al. Highly efficient non-rare-earth red emitting phosphor for warm white light-emitting diodes. Nat Commun 2014; 5: 4312 .

73 Meyer J, Tappe F. Photoluminescent materials for solid-state lighting: state of the art and future challenges. Adv Opt Mater 2015; 3: 424-430.

74 Liu ZY, Liu S, Wang K, Luo XB. Measurement and numerical studies of optical properties of YAG: Ce phosphor for white light-emitting diode packaging. App/ Opt 2010; 49: 247-257.

75 Vos WL, Tukker TW, Mosk AP, Lagendijk A, IJzerman WL. Broadband mean free path of diffuse light in polydisperse ensembles of scatterers for white light-emitting diode lighting. Appl Opt 2013; 52: 2602-2609.

76 Leung VYF, Lagendijk A, Tukker TW, Mosk AP, IJzerman WL et al. Interplay between multiple scattering, emission, and absorption of light in the phosphor of a white lightemitting diode. Opt Express 2014; 22: 8190-8204.

77 Oh JH, Yang SJ, Do YR. Healthy, natural, efficient and tunable lighting: four-package white LEDs for optimizing the circadian effect, color quality and vision performance. Light Sci App/ 2014; 3: e141.

78 Frimmer M, Chen Y, Koenderink AF. Scanning emitter lifetime imaging microscopy for spontaneous emission control. Phys Rev Lett 2011; 107: 123602.

79 Guo K, Lozano G, Verschuuren MA, Gómez-Rivas J. Control of the externa photoluminescent quantum yield of emitters coupled to nanoantenna phased arrays. J Appl Phys 2015; 118: 073103.

80 Barnes WL. Electromagnetic crystals for surface plasmon polaritons and the extraction of light from emissive devices. J Lightwave Technol 1999; 17: 2170-2182.

81 Khurgin JB, Sun G, Soref RA. Enhancement of luminescence efficiency using surface plasmon polaritons: figures of merit. J Opt Soc Am B 2007; 24: 1968-1980.

82 Sun G, Khurgin JB, Soref RA. Plasmonic light-emission enhancement with isolated metal nanoparticles and their coupled arrays. J Opt Soc Am B 2008; 25: 1748-1755.

83 Paiella R. Tunable surface plasmons in coupled metallo-dielectric multiple layers for light-emission efficiency enhancement. Appl Phys Lett 2005; 87: 111104.

84 Kelly KL, Coronado E, Zhao LL, Schatz GC. The optical properties of metal nanoparticles: the influence of size, shape, and dielectric environment. J Phys Chem B 2003; 107: 668-677.

85 Song JH, Atay T, Shi SF, Urabe H, Nurmikko AV. Large enhancement of fluorescence efficiency from CdSe/ZnS quantum dots induced by resonant coupling to spatially controlled surface plasmons. Nano Lett 2005; 5: 1557-1561.

86 Tam F, Goodrich GP, Johnson BR, Halas NJ. Plasmonic enhancement of molecular fluorescence. Nano Lett 2007; 7: 496-501.

87 Giannini V, Sánchez-Gil JA, Muskens OL, Rivas JG. Electrodynamic calculations of spontaneous emission coupled to metal nanostructures of arbitrary shape: nanoantenna-enhanced fluorescence. J Opt Soc Am B 2009; 26: 1569-1577.

88 Mertens H, Polman A. Strong luminescence quantum-efficiency enhancement near prolate metal nanoparticles: dipolar versus higher-order modes. J Appl Phys 2009; 105: 044302.

89 Bharadwaj $\mathrm{P}$, Novotny L. Plasmon-enhanced photoemission from a single $\mathrm{Y}_{3} \mathrm{~N} @ \mathrm{C}_{80}$ fullerene. J Phys Chem C 2010; 114: 7444-7447.

90 Wenger J. Fluorescence enhancement factors on optical antennas: enlarging the experimental values without changing the antenna design. Int J Opt 2012; 2012: 828121.

91 Carron KT, Fluhr W, Meier M, Wokaun A, Lehmann HW. Resonances of twodimensional particle gratings in surface-enhanced Raman scattering. J Opt Soc Am B 1986; 3: 430-440.

92 Markel VA. Antisymmetrical optical states. J Opt Soc Am B 1995; 12: 1783-1791.

93 Zou SL, Schatz GC. Narrow plasmonic/photonic extinction and scattering line shapes for one and two dimensional silver nanoparticle arrays. J Chem Phys 2004; 121 $12606-12612$.

94 Hicks EM, Zou SL, Schatz GC, Spears KG, Van Duyne RP et al. Controlling plasmon line shapes through diffractive coupling in linear arrays of cylindrical nanoparticles fabricated by electron beam lithography. Nano Lett 2005; 5: 1065-1070.

95 Chu YZ, Schonbrun E, Yang T, Crozier KB. Experimental observation of narrow surface plasmon resonances in gold nanoparticle arrays. Appl Phys Lett 2008; 93: 181108.

96 Auguié B, Barnes WL. Collective resonances in gold nanoparticle arrays. Phys Rev Lett 2008; 101: 143902.

97 Kravets VG, Schedin F, Grigorenko AN. Extremely narrow plasmon resonances based on diffraction coupling of localized plasmons in arrays of metallic nanoparticles. Phys Rev Lett 2008; 101: 087403

98 Vecchi G, Giannini V, Rivas JG. Surface modes in plasmonic crystals induced by diffractive coupling of nanoantennas. Phys Rev B 2009; 80: 201401.

99 Rodriguez SRK, Abass A, Maes B, Janssen OTA, Vecchi G et al. Coupling bright and dark plasmonic lattice resonances. Phys Rev X 2011; 1: 021019.

100 Zhou W, Odom TW. Tunable subradiant lattice plasmons by out-of-plane dipolar interactions. Nat Nanotechnol 2011; 6: 423-427.

101 Rodriguez SRK, Schaafsma MC, Berrier A, Gómez-Rivas J. Collective resonances in plasmonic crystals: size matters. Physica B 2012; 407: 4081-4085.

102 Teperik TV, Degiron A. Design strategies to tailor the narrow plasmon-photonic resonances in arrays of metallic nanoparticles. Phys Rev B 2012; 86: 245425.

103 Abass A, Rodriguez SRK, Rivas JG, Maes B. Tailoring dispersion and Eigenfield profiles of plasmonic surface lattice resonances. ACS Photon 2014; 1: 61-68.

104 de Abajo FJG. Colloquium: light scattering by particle and hole arrays. Rev Mod Phys 2007; 79: 1267-1290.

105 Christ A, Tikhodeev SG, Gippius NA, Kuhl J, Giessen H. Waveguide-plasmon polaritons: strong coupling of photonic and electronic resonances in a metallic photonic crystal slab. Phys Rev Lett 2003; 91: 183901.
106 Zentgraf T, Zhang S, Oulton RF, Zhang X. Ultranarrow coupling-induced transparency bands in hybrid plasmonic systems. Phys Rev B 2009; 80: 195415.

107 Rodriguez SRK, Murai S, Verschuuren MA, Rivas JG. Light-emitting waveguideplasmon polaritons. Phys Rev Lett 2012; 109: 166803.

108 Murai S, Verschuuren MA, Lozano G, Pirruccio G, Rodriguez SRK et al. Hybrid plasmonic-photonic modes in diffractive arrays of nanoparticles coupled to lightemitting optical waveguides. Opt Express 2013; 21: 4250-4262.

109 Pellegrini G, Mattei G, Mazzoldi P. Nanoantenna arrays for large-area emission enhancement. J Phys Chem C 2011; 115: 24662-24665.

110 Shi L, Hakala TK, Rekola HT, Martikainen JP, Moerland RJ et al. Spatial coherence properties of organic molecules coupled to plasmonic surface lattice resonances in the weak and strong coupling regimes. Phys Rev Lett 2014; 112: 153002.

111 Shi L, Yuan XW, Zhang YF, Hakala TK, Yin SY et al. Coherent fluorescence emission by using hybrid photonic-plasmonic crystals. Laser Photon Rev 2014; 8: 717-725.

112 Lozano G, Barten T, Grzela G, Rivas JG. Directional absorption by phased arrays of plasmonic nanoantennae probed with time-reversed Fourier microscopy. New J Phys 2014; 16: 013040.

113 Wiesmann C, Bergenek K, Linder N, Schwarz UT. Photonic crystal LEDs-designing light extraction. Laser Photon Rev 2009; 3: 262-286.

114 Matioli E, Weisbuch C. Impact of photonic crystals on LED light extraction efficiency: approaches and limits to vertical structure designs. J Phys D Appl Phys 2010; 43: 354005.

115 David A, Benisty H, Weisbuch C. Photonic crystal light-emitting sources. Rep Prog Phys 2012; 75: 126501.

116 Kim DH, Cho CO, Roh YG, Jeon H, Park YS et al. Enhanced light extraction from GaNbased light-emitting diodes with holographically generated two-dimensional photonic crystal patterns. Appl Phys Lett 2005; 87: 203508.

117 Gong HB, Hao XP, Wu YZ, Cao BQ, Xia W et al. Enhanced light extraction from GaNbased LEDs with a bottom-up assembled photonic crystal. Mater Sci Eng B 2011; 176: 1028-1031.

118 Lawrence N, Trevino J, Dal Negro L. Aperiodic arrays of active nanopillars for radiation engineering. J App/ Phys 2012; 111: 113101.

119 Wierer Jr JJ, David A, Megens MM. III-nitride photonic-crystal light-emitting diodes with high extraction efficiency. Nat Photon 2009; 3: 163-169.

120 Matioli E, Brinkley S, Kelchner KM, Hu YL, Nakamura S et al. High-brightness polarized light-emitting diodes. Light Sci App/ 2012; 1: e22.

121 Ding P, Li MY, He JN, Wang JQ, Fan CZ et al. Guided mode caused by silicon nanopillar array for light emission enhancement in color-converting LED. Opt Express 2015; 23: 21477-21489.

122 Okamoto K, Niki I, Shvartser A, Narukawa Y, Mukai T et al. Surface-plasmonenhanced light emitters based on InGaN quantum wells. Nat Mater 2004; 3: 601-605.

123 Gu XF, Qiu T, Zhang WJ, Chu PK. Light-emitting diodes enhanced by localized surface plasmon resonance. Nanoscale Res Lett 2011; 6: 199

124 Chu WH, Chuang YJ, Liu CP, Lee PI, Hsu SLC. Enhanced spontaneous light emission by multiple surface plasmon coupling. Opt Express 2010; 18: 9677-9683.

125 Barnes WL. Fluorescence near interfaces: the role of photonic mode density. J Mod Opt 1998; 45: 661-669.

126 DiMaria J, Dimakis E, Moustakas TD, Paiella R. Plasmonic off-axis unidirectional beaming of quantum-well luminescence. Appl Phys Lett 2013; 103: 251108.

127 Fujii T, Gao Y, Sharma R, Hu EL, DenBaars SP, Nakamura S. Increase in the extraction efficiency of GaN-based light-emitting diodes via surface roughening. Appl Phys Lett 2004; 84: 855-857.

128 Hecker NE, Höpfel RA, Sawaki N, Maier T, Strasser G. Surface plasmon-enhanced photoluminescence from a single quantum well. Appl Phys Lett 1999; 75: 1577-1579.

129 Henson J, DiMaria J, Dimakis E, Moustakas TD, Paiella R. Plasmon-enhanced light emission based on lattice resonances of silver nanocylinder arrays. Opt Lett 2012; 37: 79-81.

130 Sadi T, Oksanen J, Tulkki J. Effect of plasmonic losses on light emission enhancement in quantum-wells coupled to metallic gratings. J Appl Phys 2013; 114: 223104.

131 David A, Fujii T, Matioli E, Sharma R, Nakamura S et al. GaN light-emitting diodes with Archimedean lattice photonic crystals. Appl Phys Lett 2006; 88: 073510.

132 Giannini V, Vecchi G, Rivas JG. Lighting up multipolar surface plasmon polaritons by collective resonances in arrays of nanoantennas. Phys Rev Lett 2010; 105: 266801.

133 Teperik T, Degiron A. Control of plasmonic crystal light emission. J Opt Soc Am B 2014; 31: 223-228.

134 Rodriguez SRK, Arango FB, Steinbusch TP, Verschuuren MA, Koenderink AF et al. Breaking the symmetry of forward-backward light emission with localized and collective magnetoelectric resonances in arrays of pyramid-shaped aluminum nanoparticles. Phys Rev Lett 2014; 113: 247401.

135 Jin P, Tazawa M, Xu G. Reversible tuning of surface plasmon resonance of silver nanoparticles using a thermochromic matrix. J App/ Phys 2006; 99: 096106.

136 Olcum S, Kocabas A, Ertas G, Atalar A, Aydinli A. Tunable surface plasmon resonance on an elastomeric substrate. Opt Express 2009; 17: 8542-8547.

137 Beeckman J, Neyts K, Vanbrabant PJM. Liquid-crystal photonic applications. Opt Eng 2011; 50: 081202 .

138 Lumdee C, Toroghi S, Kik PG. Post-fabrication voltage controlled resonance tuning of nanoscale plasmonic antennas. ACS Nano 2012; 6: 6301-6307.

139 Müller J, Sönnichsen C, von Poschinger H, von Plessen G, Klar TA et al. Electrically controlled light scattering with single metal nanoparticles. Appl Phys Lett 2002; 81: $171-173$. 
140 Kossyrev PA, Yin AJ, Cloutier SG, Cardimona DA, Huang DH et al. Electric field tuning of plasmonic response of nanodot array in liquid crystal matrix. Nano Lett 2005; 5: 1978-1981.

141 Chu KC, Chao CY, Chen YF, Wu YC, Chen CC. Electrically controlled surface plasmon resonance frequency of gold nanorods. Appl Phys Lett 2006; 89: 103107.

142 Evans PR, Wurtz GA, Hendren WR, Atkinson R, Dickson W et al. Electrically switchable nonreciprocal transmission of plasmonic nanorods with liquid crystal. App/ Phys Lett 2007; 91: 043101.

143 Dickson W, Wurtz GA, Evans PR, Pollard RJ, Zayats AV. Electronically controlled surface plasmon dispersion and optical transmission through metallic hole arrays using liquid crystal. Nano Lett 2008; 8: 281-286.

144 Khatua S, Chang WS, Swanglap P, Olson J, Link S. Active modulation of nanorod plasmons. Nano Lett 2011; 11: 3797-3802.

$145 \mathrm{Li} \mathrm{HB}, \mathrm{Xu}$ SP, Gu YJ, Wang K, Xu WQ. Active modulation of wavelength and radiation direction of fluorescence via liquid crystal-tuned surface plasmons. Appl Phys Lett 2013; 102: 051107

146 Abbas A, Rodriguez SRK, Ako T, Aubert T, Verschuuren MA et al. Active liquid crystal tuning of metallic nanoantenna enhanced light emission from colloidal quantum dots. Nano Lett 2014; 14: 5555-5560

147 Bellessa J, Bonnand C, Plenet JC, Mugnier J. Strong coupling between surface plasmons and excitons in an organic semiconductor. Phys Rev Lett 2004; 93: 036404.

148 Hakala TK, Toppari JJ, Kuzyk A, Pettersson M, Tikkanen H et al. Vacuum rabi splitting and strong-coupling dynamics for surface-plasmon polaritons and rhodamine 6G molecules. Phys Rev Lett 2009; 103: 053602.

149 González-Tudela A, Huidobro PA, Martín-Moreno L, Tejedor C, García-Vidal FJ. Theory of strong coupling between quantum emitters and propagating surface plasmons. Phys Rev Lett 2013; 110: 126801.

150 Dintinger J, Klein S, Bustos F, Barnes WL, Ebbesen TW. Strong coupling between surface plasmon-polaritons and organic molecules in subwavelength hole arrays. Phys Rev B 2005; 71: 035424.

151 Vasa P, Pomraenke R, Schwieger S, Mazur YI, Kunets V et al. Coherent excitonsurface-plasmon-polariton interaction in hybrid metal-semiconductor nanostructures. Phys Rev Lett 2008; 101: 116801.

152 Schwartz T, Hutchison JA, Genet C, Ebbesen TW. Reversible switching of ultrastrong light-molecule coupling. Phys Rev Lett 2011; 106: 196405.

153 Sugawara Y, Kelf TA, Baumberg JJ, Abdelsalam ME, Bartlett PN. Strong coupling between localized plasmons and organic excitons in metal nanovoids. Phys Rev Lett 2006; 97: 266808.

154 Cade NI, Ritman-Meer T, Richards D. Strong coupling of localized plasmons and molecular excitons in nanostructured silver films. Phys Rev B 2009; 79: 241404.

155 Manjavacas A, de Abajo FJG, Nordlander P. Quantum plexcitonics: strongly interacting plasmons and excitons. Nano Lett 2011; 11: 2318-2323.

156 Zengin G, Wersäll M, Nilsson S, Antosiewicz TJ, Käll M et al. Realizing strong lightmatter interactions between single-nanoparticle plasmons and molecular excitons at ambient conditions. Phys Rev Lett 2015; 114: 157401.

157 Rodriguez SRK, Feist J, Verschuuren MA, Vidal FJG, Rivas JG. Thermalization and cooling of plasmon-exciton polaritons: towards quantum condensation. Phys Rev Lett 2013; 111: 166802 .
158 Väkeväinen AI, Moerland RJ, Rekola HT, Eskelinen AP, Martikainen JP et al. Plasmonic surface lattice resonances at the strong coupling regime. Nano Lett 2014; 14: 1721-1727.

159 Rodriguez SRK, Rivas JG. Surface lattice resonances strongly coupled to Rhodamine $6 \mathrm{G}$ excitons: tuning the plasmon-exciton-polariton mass and composition. Opt Express 2013; 21: 27411-27421.

160 Törmä P, Barnes WL. Strong coupling between surface plasmon polaritons and emitters: a review. Rep Prog Phys 2015; 78: 013901.

161 Penzkofer A, Leupacher W. Fluorescence behaviour of highly concentrated rhodamine 6G solutions. J Luminesc 1987; 37: 61-72.

162 Shen YC, Mueller GO, Watanabe S, Gardner NF, Munkholm A et al. Auger recombination in InGaN measured by photoluminescence. Appl Phys Lett 2007; 91: 141101.

163 Verzellesi G, Saguatti D, Meneghini M, Bertazzi F, Goano M et al. Efficiency droop in InGaN/GaN blue light-emitting diodes: physical mechanisms and remedies. J Appl Phys 2013; 14: 071101

164 Iveland J, Martinelli L, Peretti J, Speck JS, Weisbuch C. Direct measurement of auger electrons emitted from a semiconductor light-emitting diode under electrical injection: identification of the dominant mechanism for efficiency droop. Phys Rev Lett 2013; 110: 177406

165 Wierer JJ, Tsao JY, Sizov DS. Comparison between blue lasers and light-emitting diodes for future solid-state lighting. Laser Photon Rev 2013; 7: 963-993.

166 Meier M, Mekis A, Dodabalapur A, Timko A, Slusher RE et al. Laser action from twodimensional distributed feedback in photonic crystals. Appl Phys Lett 2009; 74: 7-9.

167 Painter O, Lee RK, Scherer A, Yariv A, O'Brien JD et al. Two-dimensional photonic band-gap defect mode laser. Science 1999; 284: 1819-1821.

168 Altug H, Englund D, Vučković J. Ultrafast photonic crystal nanocavity laser. Nat Phys 2006; 2: 484-488.

169 Hirose K, Liang Y, Kurosaka Y, Watanabe A, Sugiyama T et al. Watt-class high-power, high-beam-quality photonic-crystal lasers. Nat Photon 2014; 8: 406-411.

170 Oulton RF, Sorger VJ, Zentgraf T, Ma RM, Gladden C et al. Plasmon lasers at deep subwavelength scale. Nature 2009; 461: 629-632.

171 Stehr J, Crewett J, Schindler F, Sperling R, von Plessen G et al. A low threshold polymer laser based on metallic nanoparticle gratings. Adv Mater 2003; 15: 1726-1729.

172 van Beijnum F, van Veldhoven PJ, Geluk EJ, de Dood MJA, Hooft GW et al. Surface plasmon lasing observed in metal hole arrays. Phys Rev Lett 2013; 110: 206802.

173 Suh JY, Kim CH, Zhou W, Huntington MD, Co DT et al. Plasmonic bowtie nanolaser arrays. Nano Lett 2012; 12: 5769-5774.

174 Schokker AH, Koenderink AF. Lasing at the band edges of plasmonic lattices. Phys Rev B 2014; 90: 155452.

175 Yang AK, Hoang TB, Dridi M, Deeb C, Mikkelsen MH et al. Real-time tunable lasing from plasmonic nanocavity arrays. Nat Commun 2015; 6: 6939.

(c) (i) $\ominus$ This work is licensed under a Creative Commons Attributioncc. No ND NonCommercial-NoDerivs 4.0 International License. The images or other third party material in this article are included in the article's Creative Commons license, unless indicated otherwise in the credit line; if the material is not included under the Creative Commons license, users will need to obtain permission from the license holder to reproduce the material. To view a copy of this license, visit http:// creativecommons.org/licenses/by-nc-nd/4.0/ 